\title{
Effects of Acute and Chronic Boysenberry Intake on Blood Pressure and Endothelial Function in Spontaneous Hypertensive Rats
}

\author{
Akito MAtsushima ${ }^{1}$, Ryo FuruUchi ${ }^{1}$, Marie SHIRAI $^{2}$, Satoshi NAGAI ${ }^{2}$, Tadayuki YokOYAmA ${ }^{1}$, \\ Hiroshi NishidA ${ }^{1}$ and Masao HIRAYAMA ${ }^{1,2, *}$ \\ ${ }^{1}$ Bourbon Institutes of Health, Bourbon Corp., 4-2-14 Matsunami, Kashiwazaki, \\ Niigata 945-8611, Japan \\ ${ }^{2}$ Faculty of Applied Life Sciences, Niigata University of Pharmacy and Applied Life Sciences, \\ 265-1 Higashijima, Akiha-ku, Niigata, Niigata 956-8603, Japan
}

(Received July 16, 2013)

\begin{abstract}
Summary The effects of acute or chronic intake of boysenberry juice or artificial vinegar on blood pressure (BP) and endothelial function were investigated in spontaneous hypertensive rats (SHR). A single administration of boysenberry juice (BJ, equivalent to $0.5 \mathrm{~mL} / \mathrm{kg}$ body weight) or artificial boysenberry juice vinegar (BJV, equivalent to $0.5 \mathrm{~mL} \mathrm{BJ}$ and $0.10 \mathrm{~g}$ acetic acid/kg body weight) decreased both systolic blood pressure (SBP) and diastolic blood pressure (DBP) significantly. Reductions in SBP of the control group compared with the BJ and BJV groups reached maxima of $-16.8 \pm 4.3$ and $-28.4 \pm 7.3 \mathrm{mmHg} 8 \mathrm{~h}$ after administration, respectively. Chronic SBP- and DBP-lowering effects were also observed upon daily feedings of both BJ and BJV at 4 wk. No significant differences were found in SBP or DBP between respective acute and chronic intake of BJ and BJV, except for the decrease in DBP after 4 wk of BJV intake. This suggests that the polyphenol constituents in BJ and BJV likely play a major role in lowering SBP and DBP under these conditions and that acetic acid added to BJ exerts a DBP-lowering effect after $4 \mathrm{wk}$ of BJV intake. The polyphenolic constituents of these beverages might elevate plasma NO concentration via aortic endothelial nitric oxide synthase activation, but the effects of chronic intake on blood pressure might also be at least partly mediated by the renin-angiotensin system. These results may help explain the beneficial effects of boysenberry intake on cardiovascular health, such as reduced blood pressure and improved endothelial function.
\end{abstract}

Key Words boysenberry juice, blood pressure, endothelial function, eNOS

The juices of berries, including blackcurrant, blueberry, cranberry, and strawberry, are commonly consumed worldwide. Their major polyphenolic components are anthocyanins, which have shown a broad spectrum of biomedical effects, such as reducing cardiovascular disease (CVD), reducing oxidative stress associated with advancing age, modulating inflammatory responses, and ameliorating diverse degenerative diseases (1). Furthermore, berries contain another subclass of polyphenols, proanthocyanidins, also called flavanols or condensed tannins (2). For example, proanthocyanidins from the seeds of another fruit, cacao, have become known for health-promoting properties including reduction of CVD risk. Both the acute and chronic effects of

\footnotetext{
*To whom correspondence should be addressed.

E-mail: hirayama@nupals.ac.jp

Abbreviations: AA, acetic acid; BJ, boysenberry juice; BJC, boysenberry juice concentrate; BJV, artificial boysenberry juice vinegar; BP, blood pressure; DBP, diastolic blood pressure; eNOS, endothelial nitric oxide synthase; pACE, plasma angiotensin-converting enzyme; $\mathrm{PPh}$, polyphenol; PRA, plasma renin activity; SBP, systolic blood pressure; SHR, spontaneous hypertensive rat.
}

chocolate or cocoa proanthocyanidins on flow-mediated dilatation have been assessed in a recent meta-analysis of more than 20 randomized trials of the potential cardiovascular benefits of cocoa proanthocyanidins (3). However, the relationship between the intake of many other proanthocyanidin-rich foods and CVD risk reduction has not yet been brought to light.

Dietary proanthocyanidins have diverse structural features that vary according to their origin (4). The beverages and products of many kinds of berries, such as blueberry, blackcurrant, cranberry, chokeberry, and strawberry, contain distinctive proanthocyanidin constituents (2). We recently reported on the structural identification (5) and antihypertensive effect of polyphenols from boysenberry fruit (6). Boysenberry, a hybrid Rubus berry likely derived from a cross between Rubus loganobaccus and Rubus baileyanus Britt. (7), is cultivated in many countries. Boysenberry fruit, which is used in a number of different forms in the human diet, contains distinctive subclasses of polyphenol constituents, including anthocyanin (7) and proanthocyanidin (5). In particular, we found that boysenberry proanthocyanidins exist as short oligomers. This property is important, because only monomeric (8) and dimeric (9) proantho- 
Matsushima A et al.

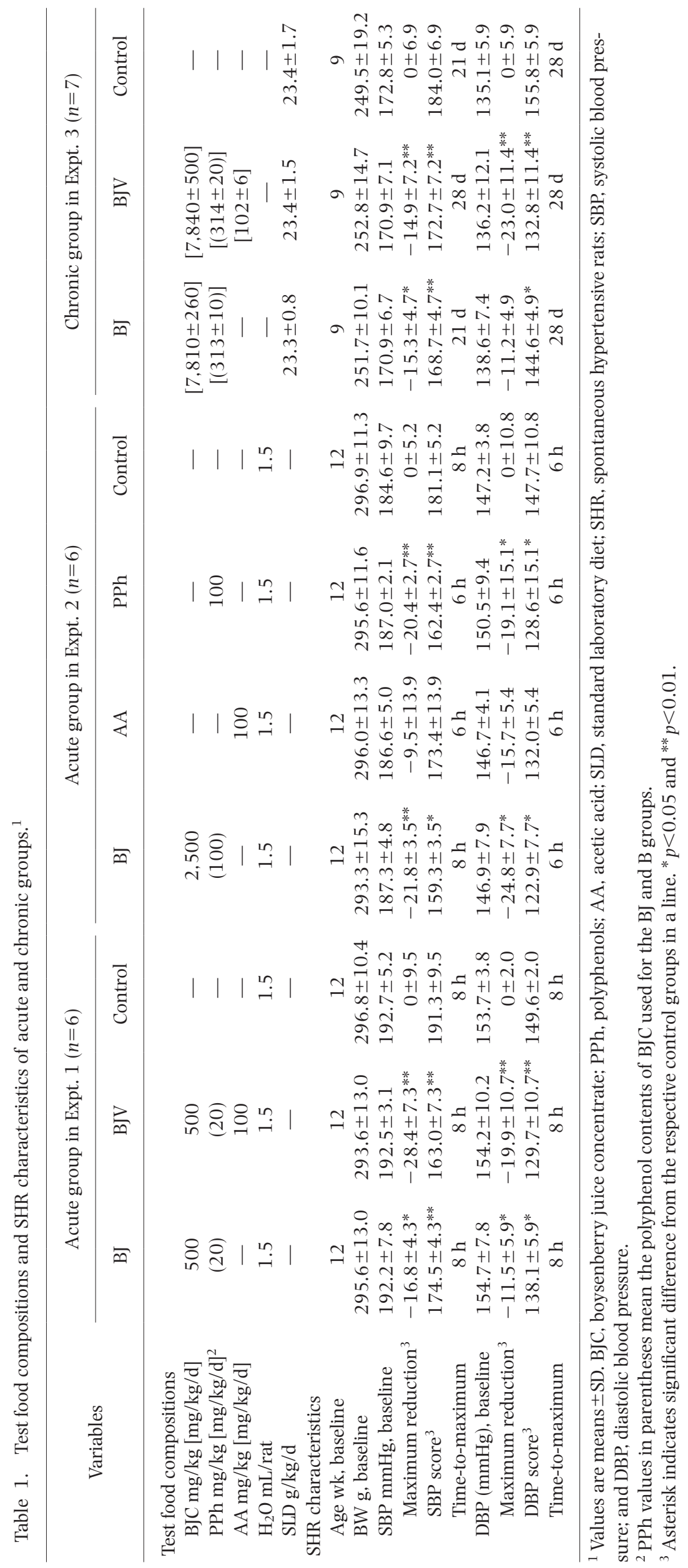


cyanidins are absorbable and bioavailable in humans. In fact, we also confirmed that a boysenberry proanthocyanidin extract exerted an in vitro vasodilative effect on the rat aortic endothelium, as well as an in vivo acute antihypertensive effect on systolic blood pressure (SBP) in spontaneous hypertensive rats (SHR) (6).

Berry vinegars, produced from the juice, are also consumed worldwide. In Japan, rice vinegar has long been an important food ingredient and is thought to be healthful, particularly for preventing hypertension. The component that is clinically active in mediating this effect was found to be acetic acid (AA), which exerts a lowering effect on BP via the renin-angiotensin system in SHR (10) and enhances flow-mediated vasodilation via endothelial nitric oxide synthase (eNOS) activation (11). Vinegar-containing beverages have now been approved by the Japanese government as foods for specified health uses (FOSHU) with demonstrated risk reduction effects on hypertension (12).

These results suggest that intake of boysenberry juice containing anthocyanins and absorbable short oligomeric proanthocyanidins exerts a BP-lowering effect and an aortic endothelial function modulating effect, which might be enhanced by the addition of AA to the juice. Therefore, we aimed to evaluate the acute and chronic effects of boysenberry juice and artificial vinegar intake on BP and endothelial function in the SHR, a common model for human hypertension.

\section{MATERIALS AND METHODS}

Rats and boysenberry food additives tested. Male SHR, purchased from Japan SLC, Inc. (Shizuoka, Japan), were housed at $24 \pm 1^{\circ} \mathrm{C}$ with a 12 -h light : dark cycle and all rats were allowed ad libitum access to commercial feed (Labo MR Stock, Nosan Co., Kanagawa, Japan) and distilled water for more than $1 \mathrm{wk}$ prior to the experiment. The study was conducted in accordance with the Animal Experimentation Guidelines of Niigata University of Pharmacy and Applied Life Sciences. Commercial boysenberry juice concentrate (BJC, $65^{\circ}$ Brix), produced by approximately 5-fold concentration of undiluted juice, was obtained from Berryfruit Export New Zealand, Ltd. (Nelson, New Zealand). Artificial boysenberry juice vinegar (BJV) was prepared by mixing BJC with AA (Kanto Chemical Co., Inc., Tokyo, Japan). Boysenberry juice polyphenol extract ( $\mathrm{PPh}$ ) was prepared from BJC and the polyphenol content was analyzed by the previously published method (5). Test foods used for acute and chronic BJ or BJV intake experiments are shown in Table 1.

Food treatments and feeding regimens. Three experiments consisting of acute intake (Experiments 1 and 2) and 4-wk (chronic) intake (Experiment 3) of test foods were performed. Numbers and baseline characteristics of SHR are shown in Table 1. Two acute treatments were performed through a single-dose administration of each test food in water $(1.5 \mathrm{~mL})$ using a stomach tube. Experiment 1 compared the BP-lowering effects among two boysenberry beverages, BJ and BJV, and water (control). Experiment 2 tested the effects of the ingredients $\mathrm{PPh}$ and AA, as positive controls for the elucidation of the active component with an effect on BP. A separate subset of Experiment 2, Experiment 2', was carried out and the rats were sacrificed by drawing blood from the abdominal aorta under ether anesthesia to test acute effects of each beverage on plasma NOx levels, reninangiotensin system activities, and aortic eNOS level. Experiment 3 compared the chronic effects of 4-wk ad libitum intake among groups of rats consuming BJ, BJV, or the control (SLD, standard laboratory diet of Labo MR stock) commercially purchased from Nihon Nosan Kogyo KK (Tokyo, Japan).

Blood pressure measurements. In acute Experiments 1 and 2, SBP and diastolic blood pressure (DBP) were measured at $0,2,4,6,8$, and $24 \mathrm{~h}$ post-administration by the tail cuff method (BP-98A-L, Softron, Tokyo, Japan). In the 4-wk chronic Experiment 3, SBP and DBP were measured at $0,1,2,3$, and 4 wk during feeding.

Plasma NOx and aortic eNOS level. After all rats in acute Experiment $2^{\prime}$ ( $6 \mathrm{~h}$ post-administration) and chronic Experiment 3 (on day 28) were anaesthetized with diethyl ether, blood $(5 \mathrm{~mL})$ was collected from the abdominal aorta with heparin-moistened syringes. Plasma was obtained by centrifugation at $2,500 \times g$ for $20 \mathrm{~min}$ at $4^{\circ} \mathrm{C}$, and stored at $-80^{\circ} \mathrm{C}$. Nitric oxide (NO) stable end products, nitrite and nitrate (NOx), were measured in the plasma using a commercial kit as described by the manufacturer (Total Nitric Oxide Assay Kit, Thermo Scientific, Rockford, IL). $\mathrm{NO}_{3}$ in plasma was converted into $\mathrm{NO}_{2}$ by reductase. Griess reagent was added to the plasma, and $\mathrm{NO}_{2}$ was converted into an azo dye compound with an absorbance peak at $540 \mathrm{~nm}$. Absorbance was then measured at $540 \mathrm{~nm}$ with a microplate reader (Model 680, Bio-Rad, Hercules, CA).

The aortic level of phosphorylated eNOS (P-eNOS) was determined by Western blotting, as previously described (13). Thoracic aortas were dissected from rats and homogenized in ice-cold lysis buffer, and the homogenates were centrifuged at $13,000 \times g$ for $5 \mathrm{~min}$. Proteins in the lysate were separated by SDS-PAGE, transferred to PVDF membranes, and probed with primary antibodies (Cell Signaling Technology, Beverly, MA) against phospho-eNOS (Ser-1177), eNOS, and GAPDH, followed by 1,000-fold dilution with Can Get Signal $^{\circledR}$ (Toyobo, Osaka, Japan) and incubation for $1 \mathrm{~h}$ at RT. After subsequent incubation with secondary antibodies (anti-rabbit IgG) conjugated with horse radish peroxidase (Cell Signaling) for $1 \mathrm{~h}$ at RT, the probed proteins were visualized using an Immobilon Western Chemiluminescent HRP Substrate (Millipore) and X-ray film (Fujifilm Co., Ltd., Tokyo, Japan). Analysis of scanned images was performed using Image Quant 5.2 software (GE Healthcare UK Ltd., Little Chalfont, UK).

Plasma renin-angiotensin system activities. Plasma renin activity (PRA) was determined according to the methods of Shah et al. (14) using a commercial kit (SensoLyte 520 Rat Renin Fluorimetric assay, AnaSpec Inc., San Jose, CA). For kinetic measurements, fluorescence intensity was measured continuously at excitation/emission wavelengths of 490/520 nm with a Gemini XPS Fluorescence Microplate Reader and SoftMax 

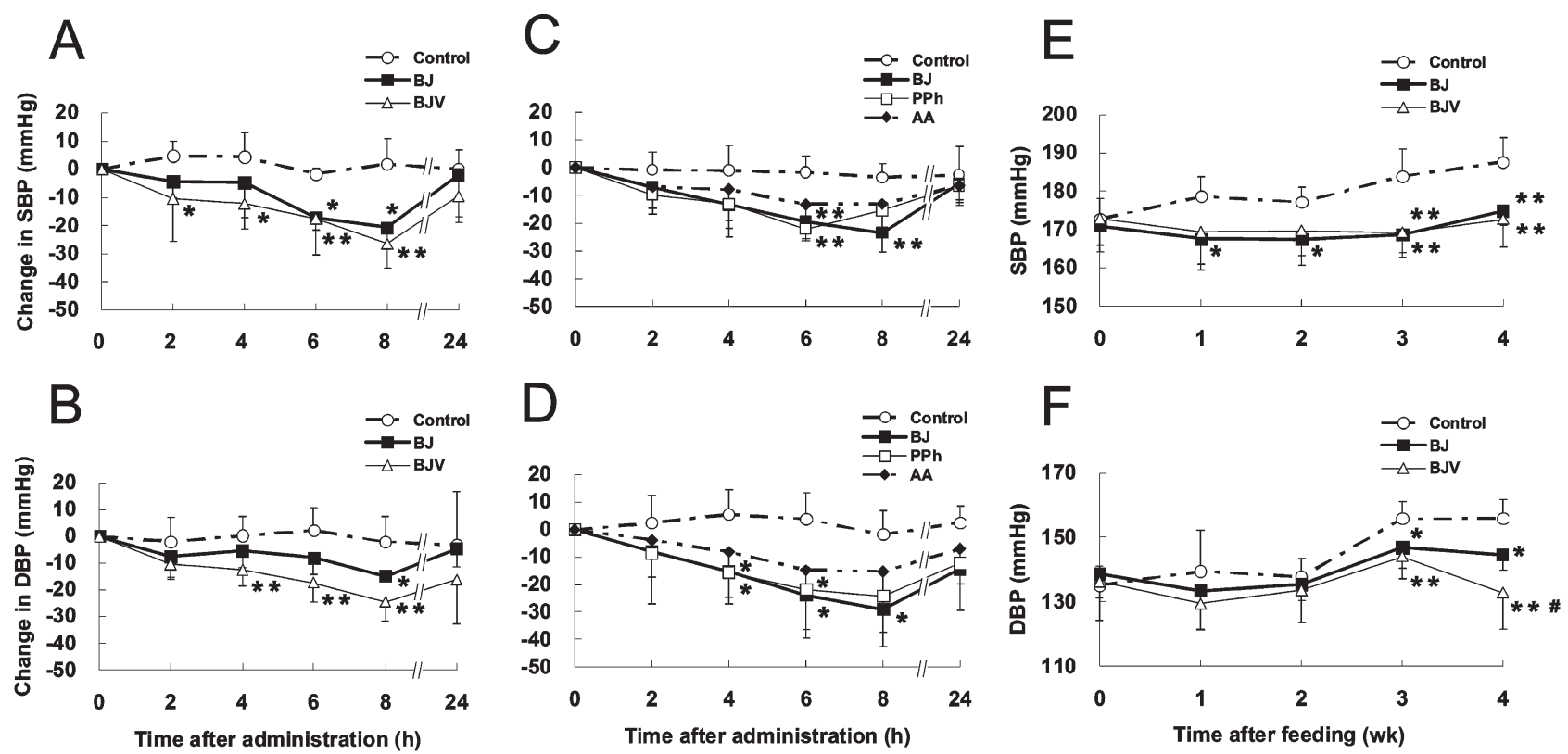

Fig. 1. Acute and chronic effects of boysenberry intake on systolic (SBP) and diastolic blood pressure (DBP) in spontaneously hypertensive rats (SHR). Acute SBP (A, C), acute DBP (B, D), chronic SBP (E), and chronic DBP (F). BJ, boysenberry juice; BJV, artificial boysenberry vinegar; AA, acetic acid; and PPh, boysenberry polyphenol extract. Results are means \pm SD of 6 acute and 7 chronic experiments. ${ }^{*} p<0.05$ and ${ }^{* *} p<0.01$ compared with respective controls at corresponding time points; ${ }^{\#} p<0.05$ compared with BJ.

Pro Software (Molecular Devices Corp., Sunnyvale, CA), and data were recorded at $1 \mathrm{~min}$ intervals for $120 \mathrm{~min}$. An end-point reading was also taken at $30 \mathrm{~min}$. The data were expressed as the rate of change in fluorescence, using the production of 5-carboxyfluorescein (5-FAM) as a marker for PRA.

Plasma angiotensin-converting enzyme (pACE) activity was determined by the modified method of Furuuchi et al. (6), using the synthetic substrate Hip-His-Leu (Sigma-Aldrich Corp., St. Louis, MO). Each plasma sample $(10 \mu \mathrm{L})$ prepared by centrifuging the blood sample was immediately incubated for $30 \mathrm{~min}$ at $37^{\circ} \mathrm{C}$ with Hip-His-Leu ( $40 \mu \mathrm{L}, 5 \mathrm{mmol} / \mathrm{L})$ in borate buffer (200 mmol/L, pH 8.3) containing $\mathrm{NaCl}(400 \mathrm{mmol} / \mathrm{L})$. After adding $1 \mathrm{~mol} / \mathrm{L} \mathrm{HCl}(190 \mu \mathrm{L})$, the mixture was centrifuged and then filtered. Each supernatant was subjected to reversed-phase HPLC analysis in an Inertsil ODS-3 column with a mobile phase of methanol-20 mmol $\mathrm{KH}_{2} \mathrm{PO}_{4}(1: 9$, pH 3.0) and detection at $228 \mathrm{~nm}$. One unit of pACE activity was defined as the amount of enzyme catalyzing release of $1 \mu \mathrm{mol} / \mathrm{L}$ per min of hippuric acid from Hip-His-Leu at $37^{\circ} \mathrm{C}$.

Plasma angiotensin II level was determined by enzyme-linked competitive immunoassay using a commercial kit (SPI Bio; Bertin Group, Montigny-le-Bretonneux, France), following the procedure recommended by the manufacturer (15). After thawing, the plasma samples were purified with Amprep Mini-Columns PH Phenyl (Amersham Biosciences, Little Chalfont, England) prior to use in plasma angiotensin II assays.

Statistical analysis. All values are presented as means \pm SD. Statistical analysis consisted of ANOVA, followed by Tukey-Kramer multiple comparison tests using
StatView 5.0 statistical software (SAS Institute Inc., Cary, NC). Values corresponding to $p<0.05$ were considered statistically significant.

\section{RESULTS}

Test foods, dosages, and rats

Table 1 presents test foods and the doses used in Experiments 1, 2, and 3. In these experiments, BJC was used as a substitute for boysenberry juice. BJC $(100 \mathrm{~g})$ contained $120 \pm 0.08 \mathrm{mg}$ polyphenols, the major components of which were $20.1 \pm 0.02 \mathrm{mg}$ anthocyanins, $0.84 \pm 0.02 \mathrm{mg}$ proanthocyanidins, and $40.18 \pm 0.02 \mathrm{mg}$ hydrolyzable tannins. Doses used for the single administration in Experiments 1 and 2 were 0.5 or $2.5 \mathrm{~g}$ BJC per kg body weight, respectively, which corresponded to consumption of 30 or $150 \mathrm{~mL}$ of undiluted boysenberry juice by a $60 \mathrm{~kg}$ human. Chronic daily intake of BJC $(7.8 \mathrm{~g} / \mathrm{kg}$ body weight per day) in Experiment 3 corresponded to consumption of three $150 \mathrm{~mL}$ cups of undiluted boysenberry juice per day by a human. SHR characteristics are shown in Table 1; the baseline SBP for rats in acute and chronic BJ intake groups ranged from $184.2 \pm 6.9$ to $192.7 \pm 5.2 \mathrm{mmHg}$ and from $170.9 \pm 6.7$ to $172.8 \pm 5.3 \mathrm{mmHg}$, respectively. During the chronic experimental period, there was no significant difference among the three groups in either their food consumption averages (SLD in Table 1) or average body weights, which reached $299.9 \pm 9.2$, $302.0 \pm 4.7$, or $298.0 \pm 4.8 \mathrm{~g}$ in BJ, BJV, or control rats, respectively, after $4 \mathrm{wk}$.

Effects of BJ or BJV on blood pressure in SHR

Acute SBP- and DBP-lowering effects were observed upon a single administration of both $\mathrm{BJ}(0.5 \mathrm{~g}$ of $\mathrm{BJC} /$ 


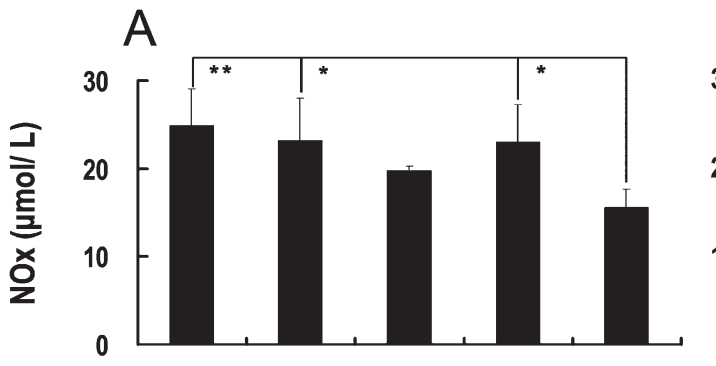

B

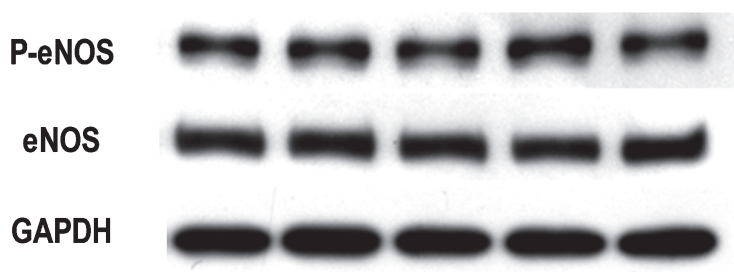

C

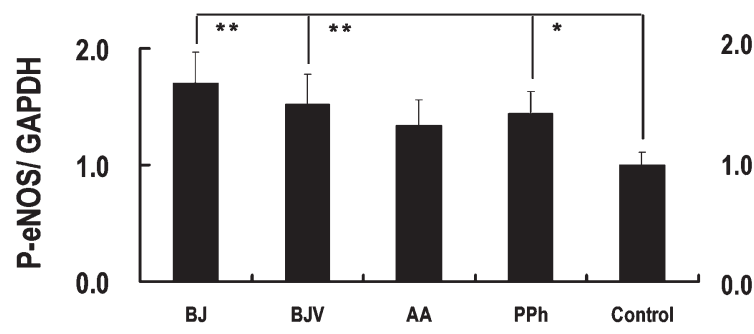

$\mathrm{D}$

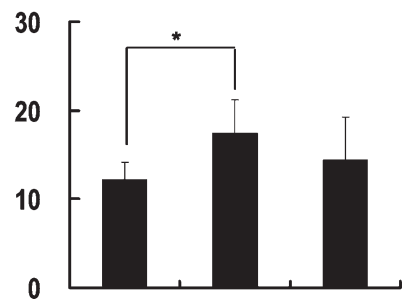

$E$

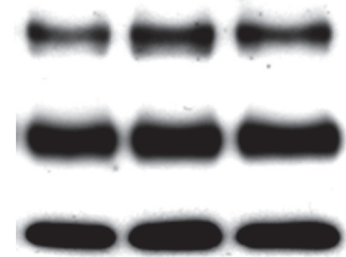

$\mathrm{F}$

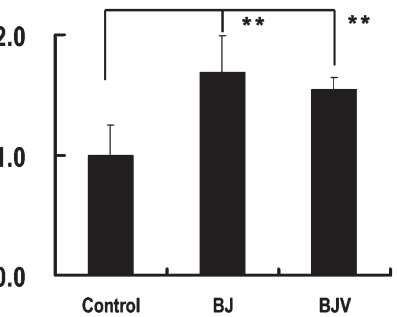

Fig. 2. Comparison of acute and chronic effects of boysenberry intake on plasma nitrate (NOx) concentration, Western blotting, and the strength of aortic phospho-eNOS (P-eNOS) in spontaneously hypertensive rats (SHR). Acute and chronic effects were measured $6 \mathrm{~h}$ after administration and $28 \mathrm{~d}$ after feeding, respectively, NOx concentration (A, D), Western blotting (B, E), and P-eNOS strength (C, F). BJ, boysenberry juice; BJV, artificial boysenberry vinegar; AA, acetic acid; and $\mathrm{PPh}$, boysenberry polyphenol extract. Bars are means \pm SD of six acute and seven chronic experiments. ${ }^{*} p<0.05$ and ${ }^{* *} p<0.01$ compared with respective controls.

$\mathrm{kg}$ body weight) and BJV to rats $(0.5 \mathrm{~g}$ of $\mathrm{BJC}$ and $0.10 \mathrm{~g}$ of AA/kg body weight) (Fig. $1 \mathrm{~A}$ and B; Table 1). $\mathrm{SBP}$ in rats under either the $\mathrm{BJ}$ or $\mathrm{BJV}$ intake regimen was significantly reduced from the control at 6-8 h or at $2-8 \mathrm{~h}$ after administration, and reached maxima of $-16.8 \pm 4.3(p<0.05)$ or $-28.4 \pm 7.3 \mathrm{mmHg}(p<0.01)$ at $8 \mathrm{~h}$, respectively. DBP changes in rats fed either BJ or BJV showed similar reduction profiles, with values reaching significance at $4-8 \mathrm{~h}$ after administration, and maxima of $-11.5 \pm 5.9$ or $-19.9 \pm 10.7 \mathrm{mmHg}$ at $8 \mathrm{~h}$, respectively. After $24 \mathrm{~h}$, the SBP and DBP changes in both groups of rats returned to levels not significantly different from the control. There were no significant differences in SBP or DBP between rats under BJ or BJV intake treatments. Experiment $2^{\prime}$ was designed to compare the effect of candidate active ingredients $\mathrm{PPh}$ and AA as positive controls in groups of rats fed BJ, PPh, AA, or control (Fig. 1C and D; Table 1). Rats fed PPh exhibited significant reductions in SBP $(-20.4 \pm 2.7 \mathrm{mmHg}$, $p<0.01)$ and DBP $(-19.1 \pm 15.1 \mathrm{mmHg}, p<0.05)$ at $6 \mathrm{~h}$ after administration compared with rats in the control group, and their BP profiles were very similar to those of rats in the $\mathrm{BJ}$ group. In contrast, rats fed AA exhibited no significant difference from the control in either SBP or DBP.

Chronic SBP- and DBP-lowering effects were also observed both in rats fed BJ daily for $4 \mathrm{wk}(7.81 \pm 0.26 \mathrm{~g}$ $\mathrm{BJC} / \mathrm{kg}$ body weight) and those fed BJV (7.84 $\pm 0.50 \mathrm{~g}$ $\mathrm{BJC}$ and $102 \pm 6 \mathrm{mg} \mathrm{AA} / \mathrm{kg}$ body weight) (Fig. 1E and F; Table 1). Rats with chronic BJ intake exhibited significantly lower SBP than the control at 1-4 wk, with BP of $168.7 \pm 4.7 \mathrm{mmHg}$ at $3 \mathrm{wk}(p<0.01)$ compared with BP of $184.0 \pm 6.9 \mathrm{mmHg}$ for the control. Rats with chronic BJV intake also had significantly lower BP at 3-4 wk, with $\mathrm{BP}$ of $172.7 \pm 7.2 \mathrm{mmHg}(p<0.01)$ at $4 \mathrm{wk}$. DBP was significantly lower in rats in both the BJ and BJV intake groups compared with the control at 3-4 wk. BJV rats showed significant BP reduction from $3 \mathrm{wk}$ to $4 \mathrm{wk}$. After comparing chronic SBP and DBP values between rats in the $\mathrm{BJ}$ and $\mathrm{BJV}$ intake groups, DBP values for rats in the BJV intake group were found to be significantly lower than those for the rats in the BJ intake group only at $4 \mathrm{wk}(p<0.05)$.

Effects of BJ or BJV on plasma NOx and aortic e-NOS levels

Acute effects of a single administration were examined in rats at $6 \mathrm{~h}$ post-administration (Fig. 2A-C) and data for chronic effects were obtained from rats after 4-wk daily feeding of treatments or controls (Fig. 

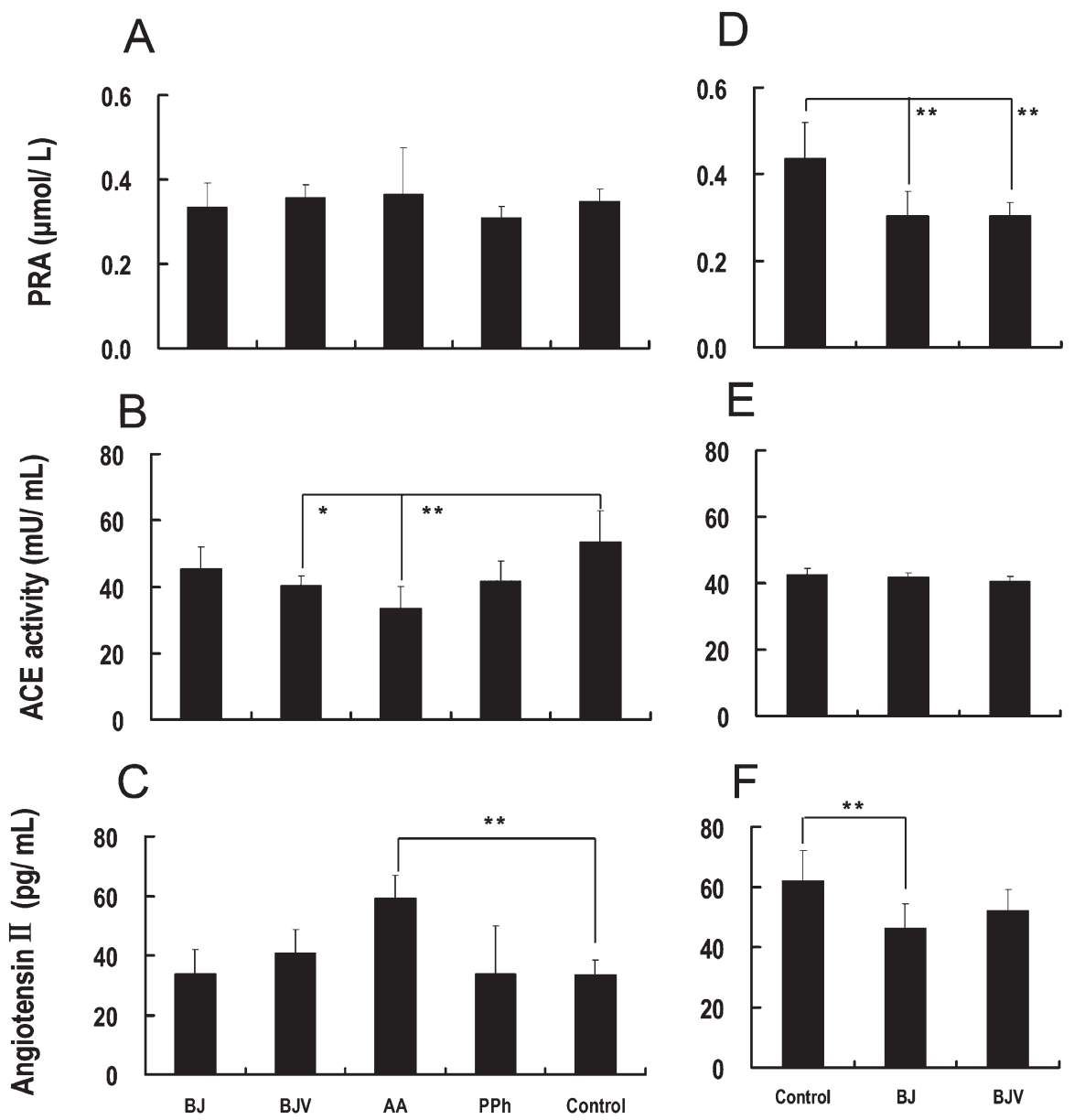

Fig. 3. Comparison of acute and chronic effects of boysenberry intake on plasma renin activity (PRA), plasma angiotensin converting enzyme (pACE) activity, and angiotensin II level in plasma in spontaneously hypertensive rats (SHR). Acute and chronic effects were measured $6 \mathrm{~h}$ after administration and $28 \mathrm{~d}$ after feeding, respectively. PRA (A, D), pACE activity (B, E), and angiotensin II level (C, F). BJ, boysenberry juice; BJV, artificial boysenberry vinegar; AA, acetic acid; and PPh, boysenberry polyphenol extract. Bars are means \pm SD of six acute and seven chronic experiments. ${ }^{*} p<0.05$ and ${ }^{* *} p<0.01$ compared with respective controls.

2D-F). Plasma NOx levels in rats from the BJ intake groups $(2.5 \mathrm{~g} \mathrm{BJC} / \mathrm{kg}$ body weight $)$ increased in both acutely $(24.9 \pm 4.2 \mu \mathrm{mol} / \mathrm{L}, p<0.01)$ and chronically $(17.4 \pm 3.8 \mu \mathrm{mol} / \mathrm{L}, p<0.05)$ administered rats, and were significantly higher than in rats in their respective control groups $(16.5 \pm 1.8$ and $14.3 \pm 4.9 \mu \mathrm{mol} / \mathrm{L})$. Similar increases were observed in rats in the BJV intake group, but a significant difference was found only for the acute rats $(23.2 \pm 4.9 \mu \mathrm{mol} / \mathrm{L}, p<0.05)$. A significant increase in acute NOx level was also observed in rats fed PPh $(22.1 \pm 3.8 \mu \mathrm{mol} / \mathrm{L}, p<0.05)$, but rats fed AA $(19.7 \pm 0.6 \mu \mathrm{mol} / \mathrm{L})$ showed no significant difference in NOx level compared with the control.

Western blot analyses of aortic levels of total eNOS, phospho-eNOS-Ser1177 (P-eNOS), and GAPDH protein were performed in acute and chronic rats (Fig. 2B and E), and all data for eNOS and P-eNOS levels were normalized with respect to GAPDH. Aortic eNOS levels were not significantly altered among respective rats in either experiment (data not shown). On the other hand, $\mathrm{P}-\mathrm{eNOS}$ levels were found to have increased significantly not only in rats under acute $\mathrm{BJ}, \mathrm{BJV}$, and $\mathrm{PPh}$ intake
(1.6-, 1.5-, 1.3-, and 1.4-fold higher than the control, respectively), but also in rats under chronic $\mathrm{BJ}$ and $\mathrm{BJV}$ intake (1.7- and 1.5-fold higher).

Effects of BJ or BJV on plasma renin-angiotensin system activities

The effects of boysenberry juice on plasma PRA, pACE activity, and angiotensin II level, were compared between the acute and chronic intake experiments (Fig. $3 \mathrm{~A}$ and $\mathrm{F})$. With regard to PRA, acute administration resulted in no significant change in the BJ, BJV, AA, or $\mathrm{PPh}$ groups compared to the control. However, chronic feeding of both BJ and BJV to rats caused significant decreases $(0.304 \pm 0.06$ and $0.303 \pm 0.03 \mu \mathrm{mol} / \mathrm{L}$, $p<0.01)$ relative to the control $(0.437 \pm 0.082 \mu \mathrm{mol} / \mathrm{L})$. There was no significant difference in pACE activity between rats fed $\mathrm{BJ}$ or $\mathrm{PPh}$ in either the acute or chronic experiments, while pACE activity decreased significantly compared to the control in rats acutely administered BJV or AA ( $p<0.05$ and $<0.01$ respectively). Angiotensin II levels significantly increased compared to control upon acute administration of AA to rats $(59.14 \pm 16.32 \mathrm{pg} / \mathrm{L}$, $p<0.01$ ), although no significant change in angiotensin 
II level was found in rats acutely administered BJ, BJV, or $\mathrm{PPh}$. However, upon chronic intake of BJ, angiotensin II levels decreased significantly $(62.2 \pm 10.0 \mathrm{pg} / \mathrm{L}$, $p<0.01)$ compared with the control $(46.3 \pm 8.15 \mathrm{pg} / \mathrm{L})$.

\section{DISCUSSION}

The present findings show clear effects of acute or chronic intake of boysenberry juice and artificial vinegar on lowering BP in SHR, and implied improvement of endothelial functions through phosphorylation of eNOS and endothelial NO production in the SHR aorta.

The boysenberry is cultivated in many countries and the juice concentrate is widely used for beverage products, such as BJ and BJV. In this study, the single oral dose in the acute study was $500 \mathrm{mg} \mathrm{BJC} / \mathrm{kg}$ body weight in rats, which corresponds to $30 \mathrm{~mL}$ of fresh $\mathrm{BJ}$ for a $60-\mathrm{kg}$ human. The intended daily dose for the chronic study was $7,500 \mathrm{mg} \mathrm{BJC} / \mathrm{kg}$ body weight per day in rats, which corresponds to a $60-\mathrm{kg}$ human consuming $150 \mathrm{~mL} \mathrm{BJ}$ three times per day.

Single administrations of BJ and BJV to SHR caused significant acute decreases in both SBP and DBP compared to the control. Comparison of the SBP and DBP responses in rats under $\mathrm{BJ}$ and $\mathrm{BJV}$ administration during a $24 \mathrm{~h}$ period revealed that the respective BP reduction profiles were similar in both treatments, with maxima occurring $8 \mathrm{~h}$ after administration (Fig. 1A and B). In a chronic 4-wk feeding experiment, rats that were administered BJ and BJV also had significantly lower SBP and DBP than control rats (Fig. 1E and F). SBP profiles were very similar for rats under BJ or BJV intake, and reached their respective reduction maxima of $-15.3 \pm 4.7$ and $-14.9 \pm 7.2 \mathrm{mmHg}$. DBP profiles also showed significant decreases in rats under both BJ and BJV intake after 3 and $4 \mathrm{wk}$. No significant difference in BP was found for rats under either acute or chronic intake of BJ or BJV, except for the observed reduction in DBP under chronic intake of BJ or BJV at $4 \mathrm{wk}$. These results indicated that acute and chronic intake of BJ and BJV lowered SBP and DBP and that the respective response patterns were very similar in both BJ and BJV intake.

These results were interesting because we had expected the BP-lowering activity to be greater for groups under BJV intake than for those under BJ intake, due to the $5 \%(\mathrm{w} / \mathrm{w})$ content of AA in BJV. AA is known as an active dietary ingredient (10) used in FOSHU with risk reduction effects on hypertension in Japan (12). We had previously reported that boysenberry juice contained considerable amounts of polyphenols including anthocyanins $(105 \mathrm{mg} / 100 \mathrm{~mL})$ and proanthocyanidins $(3.07 \mathrm{mg} / 100 \mathrm{~mL})(5)$ and that the proanthocyanidins had both in vitro vasodilating and BP-lowering effects in SHR. In acute Experiment 2', the BP-lowering effects of AA and PPh were evaluated, and SBP and DBP were shown to significantly decrease in rats administered $\mathrm{PPh}$, while the SBP and DBP of rats administered AA showed no significant change (Fig. 1C and D). In addition, time course profiles of SBP and DBP responses were similar in rats under $\mathrm{PPh}$ and $\mathrm{BJ}$ administration. Although no significant differences were found in SBP or DBP between respective groups with acute or chronic intake of BJ or BJV, there was a significant decrease in DBP after $4 \mathrm{wk}$ of BJV intake. This suggests that the polyphenol constituents, or a synergistic effect of the polyphenols and AA, in BJ and BJV likely play a major role in lowering SBP and DBP under these conditions and that the AA added to BJ exerts a DBP-lowering effect after $4 \mathrm{wk}$ of BJV intake. However, the reason for this decrease in BJV remains obscure, although there may be a synergistic component in $\mathrm{BJ}$ or $\mathrm{BJV}$ regulating $\mathrm{BP}$ because significant differences in the levels of plasma NOx and angiotensin II after 4 wk were found only in the BJ intake group and not in the BJV intake group compared to the control (Figs. 2D and 3F). However, this does not explain the observed decrease only in DBP without a corresponding decrease in SBP, which indicates the need for further investigation.

To link the BP-lowering effects of boysenberry intake with underlying endothelial functions, we evaluated plasma total NOx level to estimate NO production, eNOS protein level in the SHR aorta, and the relationship between them. Nitric oxide (NO) is a critical regulator of endothelial function (16). Dietary polyphenols, such as particular flavonoids (17), cacao proanthocyanidins (18), and olive oil extract (19) acutely augment NO status and thereby enhance endothelial function. Plasma NOx levels were significantly augmented in groups of rats acutely administered $\mathrm{BJ}(p<0.01)$, BJV $(p<0.05)$, or PPh $(p<0.05)$ compared with the control, whereas there was no significant effect on plasma NOx levels in rats administered AA $(p=0.38)$. The observed increases in NOx levels in the rat groups under BJ, BJV, AA, and $\mathrm{PPh}$ intake treatments showed good correlation with changes in aortic P-eNOS levels normalized to GAPDH and expressed relative to the control (Fig. 2C). The $p$ values for aortic P-eNOS levels in BJ, BJV, AA, and PPh intake groups compared with control were $<0.01$, $<0.01,0.127$, and 0.03, respectively. Sakakibara et al. reported that AA upregulated eNOS activity through enhancement of phosphorylation of Ser-1177 in human umbilical vein endothelial cells (11). However, rats administered AA in this study did not show significant enhancement of aortic P-eNOS activity. Rats under chronic intake of $\mathrm{BJ}$ showed significant increases in both NOx $(p=0.047)$ and P-eNOS levels $(p<0.01)$. Under BJV intake, significant effects were found for aortic P-eNOS levels $(p<0.01)$ in rats, although only non-significant effects on NOx levels ( $p=0.54$ ) were observed. Good correlations between plasma NOx level and aortic P-eNOS levels were found in both acute and chronic BJ intake groups in this study. These findings suggest that dietary BJ augmented eNOS phosphorylation and induced a subsequent increase in plasma NO. In addition, the responses to BJ intake reported here are very similar to those recently reported for acute and chronic treatments of SHR with a dietary ginseng extract (20) or statin drugs $(13,21)$.

The renin-angiotensin system plays a crucial role in the development of hypertension via an increase in renin activity and production of angiotensin II. We have 
previously shown that boysenberry proanthocyanidins are not acutely associated with plasma ACE activity in SHR (6), but the BJ used in this study contained a multitude of other uncharacterized phytochemical constituents in addition to the proanthocyanidins. Therefore, we compared the effects of boysenberry intake on plasma renin-angiotensin system activities among the five acute and three chronic treatment groups (Fig. 3A-F) in this study. There was no significant difference in responses among the acute $\mathrm{BJ}, \mathrm{PPh}$, and control intake groups for PRA, pACE activity, or plasma angiotensin II levels. These results indicate that $\mathrm{BJ}$ and $\mathrm{PPh}$ intake was not associated with acute responses in plasma renin-angiotensin system activities. The rats in the AA intake group showed a significant decrease in pACE activity $(p<0.01)$ and an increase in angiotensin II levels $(p<0.01)$, while rats in the BJV intake group showed a significant decrease in pACE activity $(p<0.05)$. These results provide support for the role of AA in acute changes in renin-angiotensin system activities, although Kondo et al. (10) also reported a significant PRA decrease upon chronic ( $8 \mathrm{wk}$ ) AA feeding. Interestingly, chronic intake of $\mathrm{BJ}$ and BJV resulted in distinct responses for PRA and plasma angiotensin II levels from those revealed under acute intake. There were no significant responses in PRA or angiotensin II activities upon acute intake, but significant decreases compared to control were observed in PRA $(p<0.01$ for both BJ and BJV) and angiotensin II levels $(p<0.01$ for BJ) upon chronic intake. The observed PRA attenuation upon BJV intake was, in part, expected because chronic intake of rice vinegar containing AA has previously been reported to attenuate PRA in SHR (10). However, we did not expect chronic BJ intake to significantly attenuate both PRA and plasma angiotensin II levels, a phenomenon that was not observed upon acute BJ intake. The reduced activities of these two biomarkers of the renin-angiotensin system were likely responsible for the observed chronic reductions in SBP and DBP, suggesting differences between the mechanisms governing the BP-lowering effects of acute and chronic BJ intake. That is, the chronic BPlowering effect of $\mathrm{BJ}$ intake might be mediated not only by endothelial NO generation, but also by the combined contribution of renin-angiotensin system activities that had no contribution to the acute effect. This is the first finding that chronic intake of berry juice may, at least in part, be associated with the plasma activities of the renin-angiotensin system in reducing BP. However, further experiments are needed to more accurately define the in vivo antihypertensive mechanisms of $\mathrm{BJ}$ intake, for example, to identify bioactive components and evaluate renin-angiotensin system activities in other organs including the aorta and kidney.

In conclusion, this study shows that acute and chronic intake of boysenberry juice and artificial boysenberry juice vinegar results in significant reduction of both SBP and DBP in SHR. Polyphenolic constituents of these beverages under both intake regimens play a distinctive role in elevating plasma NO concentration via aortic eNOS activation, but renin-angiotensin system activities might also be partly responsible for BP modulation under chronic intake. These results are relevant to improving our understanding of the beneficial effects of boysenberry intake, such as lowered blood pressure and improved endothelial function, on cardiovascular health.

\section{REFERENCES}

1) Zafra-Stone S, Yasmin T, Bagchi M, Chatterjee A, Vinson JA, Bagchi D. 2007. Berry anthocyanins as novel antioxidants in human health and disease prevention. Mol Nutr Food Res 51: 675-683.

2) Hellström JK, Törrönen AR, Mattila PH. 2009. Proanthocyanidins in common food products of plant origin. $J$ Agric Food Chem 57: 7899-7906.

3) Hooper L, Kay C, Abdelhamid A, Kroon PA, Cohn JS, Rimm EB, Cassidy A. 2012. Effects of chocolate, cocoa, and flavan-3-ols on cardiovascular health: a systematic review and meta-analysis of randomized trials. Am J Clin Nutr 95: 740-751.

4) Aherne SA, O’Brien NM. 2002. Dietary flavonols: chemistry, food content, and metabolism. Nutrition 18: 75-81.

5) Furuuchi R, Yokoyama T, Watanabe Y, Hirayama M. 2011. Identification and quantification of short oligomeric proanthocyanidins and other polyphenols in boysenberry seeds and juice. J Agric Food Chem 59: 3738-3746.

6) Furuuchi R, Sakai H, Hirokawa N, Watanabe Y, Yokoyama T, Hirayama M. 2012. Antihypertensive effect of boysenberry seed polyphenols on spontaneously hypertensive rats and identification of orally absorbable proanthocyanidins with vasorelaxant activity. Biosci Biotechnol Biochem 76: 1694-1701.

7) McGhie TK, Rowan DR, Edwards PJ. 2006. Structural identification of two major anthocyanin components of boysenberry by NMR spectroscopy. J Agric Food Chem 54: 8756-8761.

8) Roura E, Andrés-Lacueva C, Jáuregui O, Badia E, Estruch R, Izquierdo-Pulido M, Lamuela-Raventós RM. 2005. Rapid liquid chromatography tandem mass spectrometry assay to quantify plasma (-)-epicatechin metabolites after ingestion of a standard portion of cocoa beverage in humans. J Agric Food Chem 53: 6190-6194.

9) Holt RR, Lazarus SA, Sullards MC, Zhu QY, Schramm DD, Hammerstone JF, Fraga CG, Schmitz HH, Keen CL. 2002. Procyanidin dimer B2 [epicatechin-( $4 \beta-8)$-epicatechin] in human plasma after the consumption of a flavanol-rich cocoa. Am J Clin Nutr 76: 798-804.

10) Kondo S, Tayama K, Tsukamoto Y, Ikeda K, Yamori Y. 2001. Antihypertensive effects of acetic acid and vinegar on spontaneously hypertensive rats. Biosci Biotechnol Biochem 65: 2690-2694.

11) Sakakibara S, Murakami R, Takahashi M, Fushimi T, Murohara T, Kishi M, Kajimoto Y, Kitakaze M, Kaga T. 2010. Vinegar intake enhances flow-mediated vasodilatation via upregulation of endothelial nitric oxide synthase activity. Biosci Biotechnol Biochem 74: 1055-1061.

12) Yamada K, Sato-Mito N, Nagata J, Umegaki K. 2008. Health claim evidence requirements in Japan. J Nutr 138: 1192S-1198S.

13) Ohkawara $H$, Ishibashi $T$, Saitoh $S$, Inoue $N$, Sugimoto K, Kamioka M, Uekita H, Kaneshiro T, Ando K, Takuwa Y, Maruyama Y, Takeishi Y. 2010. Preventive effects of pravastatin on thrombin-triggered vascular responses 
via Akt/eNOS and RhoA/Racl pathways in vivo. Cardiovasc Res 88: 492-501.

14) Shah N, Dhar D, El Zahraa Mohammed F, Habtesion A, Davies NA, Jover-Cobos M, Macnaughtan J, Sharma V, Olde Damink SWM, Mookerjee RP, Jalan R. 2012. Prevention of acute kidney injury in a rodent model of cirrhosis following selective gut decontamination is associated with reduced renal TLR4 expression. J Hepatol 56: 1047-1053.

15) Volland H, Pradelles P, Ronco P, Azizi M, Simon D, Creminon C, Grassi J. 1999. A solid-phase immobilized epitope immunoassay (SPIE-IA) permitting very sensitive and specific measurement of angiotensin in plasma. J Iтmunol Methods 228: 37-47.

16) Kleinbongard P, Dejam A, Lauer T, Jax T, Kerber S, Gharini P, Balzer J, Zotz RB, Scharf RE, Willers R, Schechter AN, Feelisch M, Kelm M. 2006. Plasma nitrite concentrations reflect the degree of endothelial dysfunction in humans. Free Radic Biol Med 40: 295-302.

17) Loke WM, Hodgson JM, Proudfoot JM, McKinley AJ, Puddey IB, Croft KD. 2008. Pure dietary flavonoids quercetin and (-)-epicatechin augment nitric oxide products and reduce endothelin-1 acutely in healthy men. Am J
Clin Nutr 88: 1018-1025.

18) Taubert D, Roesen R, Lehmann C, Jung N, Schömig E. 2007. Effects of low habitual cocoa intake on blood pressure and bioactive nitric oxide: a randomized controlled trial. JAMA 298: 49-60.

19) Moreno-Luna R, Muñoz-Hernandez R, Miranda ML, Costa AF, Jimenez-Jimenez L, Vallejo-Vaz AJ, Muriana FJ, Villar J, Stiefel P. 2012. Olive oil polyphenols decrease blood pressure and improve endothelial function in young women with mild hypertension. Am J Hypertens 25: 1299-1304.

20) Hong SY, Kim JY, Ahn HY, Shin JH, Kwon O. 2012. Panax ginseng extract rich in ginsenoside protopanaxatriol attenuates blood pressure elevation in spontaneously hypertensive rats by affecting the Akt-dependent phosphorylation of endothelial nitric oxide synthase. J Agric Food Chem 60: 3086-3091.

21) Suh JW, Choi DJ, Chang HJ, Cho YS, Youn TJ, Chae IH, Kim KI, Kim CH, Kim HS, Oh BH, Park YB. 2010. HMGCoA reductase inhibitor improves endothelial dysfunction in spontaneous hypertensive rats via down-regulation of caveolin-1 and activation of endothelial nitric oxide synthase. J Korean Med Sci 25: 16-23. 